\title{
Multiphoton polarization and generalized polarization microscopy reveal oleic-acid-induced structural changes in intercellular lipid layers of the skin
}

\author{
Yen Sun and Wen Lo \\ Department of Physics, National Taiwan University, Taipei 106, Taiwan \\ Sung-Jan Lin \\ Department of Dermatology, National Taiwan University Hospital, Taipei 100, Taiwan \\ Shiou-Hwa Jee \\ Department of Dermatology, National Taiwan University Hospital and University College of Medicine, Taipei 100, Taiwan \\ Chen-Yuan Dong \\ Department of Physics, National Taiwan University, Taipei 106, Taiwan
}

Received March 17, 2004

\begin{abstract}
We have demonstrated that both multiphoton polarization and generalized polarization (GP) microscopy may be combined to characterize the structural changes of intercellular lipids in skin. Both polarization and GP (at 440- and 490-nm emission) images obtained by analysis of Laurdan fluorescence suggest that the treatment of oleic acid results in a skin surface with a more random packing of lipid molecules, which allows easier water penetration. Our results show that combined polarization and GP microscopy can be used to characterize the physical and chemical changes in biological structures. () 2004 Optical Society of America

OCIS codes: $190.4180,180.2520,170.1870$.
\end{abstract}

The introduction of two-photon fluorescence microscopy by Webb's group at Cornell University revolutionized biological scanning microscopy. ${ }^{1}$ Pointlike excitation volume, reduced photodamage, and enhanced imaging depths are among the advantages of this technique. In recent years the generalized technique of multiphoton microscopy has evolved into a powerful technique for investigating a wide spectrum of phenomena in the life sciences. ${ }^{2}$ A unique area in which multiphoton microscopy has made a significant contribution is in revealing the mechanisms of transdermal delivery pathways. ${ }^{3-5}$ Although it is believed that an increase of the skin's lipid fluidity is responsible for enhanced delivery, it is difficult to observe the changes in the skin's physical properties from intensity imaging alone. Other important biological processes such as endocytosis, exocytosis, and cell death also involve changes in the membrane characteristics of cells, and the development of microscopic techniques to characterize these processes have important implications in cell biology.

In the study reported in this Letter we combined two multiphoton imaging techniques for characterizing changes in the physical and chemical properties of intercellular lipid layers of the skin: polarization and generalized polarization (GP) imaging. Both techniques have been demonstrated. ${ }^{6,7}$ However, to the best of our knowledge, they have not been combined and applied to monitor the changes in biological barriers such as intercellular lipid layers of the skin. It is our intention to characterize the sensitivities of these two approaches in detecting changes in the skin's lipid structures in the presence of the chemical enhancer oleic acid (OA). Our results should be of interest for research in cell biology and biological delivery processes. The outermost layer of the epidermis is the stratum corneum, composed of cellular and intercellular regions. The intercellular domain is composed of stacks of highly structured lipid lamellae with ceramides, cholesterol, and other fatty acids as the main constituents. The intercellular lipids act as the main barrier to water loss and drug delivery. ${ }^{8,9}$

In conventional polarization spectroscopy the rotational properties of fluorescent molecules are studied by exciting the sample with polarized light. The polarized fluorescence emission is then characterized by measuring the fluorescence parallel $\left(I_{\mathrm{par}}\right)$ and perpendicular $\left(I_{\mathrm{per}}\right)$ to the excitation polarization. The values of polarization $P$ and anisotropy $r$, defined in the following equations, can then be used to characterize the state of the sample order ${ }^{10}$ :

$$
P=\frac{I_{\mathrm{par}}-I_{\mathrm{per}}}{I_{\mathrm{par}}+I_{\mathrm{per}}}, \quad r=\frac{I_{\mathrm{par}}-I_{\mathrm{per}}}{I_{\mathrm{par}}+2 I_{\mathrm{per}}}
$$

Therefore a randomly oriented sample tends to have a polarization value close to zero, whereas a highly ordered specimen tends to have a high (absolute) polarization value.

Another technique of GP has also been implemented to characterize the microenvironment of the fluorescent probe Laurdan. In this approach the GP value is an indication of the spectral shift of Laurdan and is 
defined as

$$
\mathrm{GP}=\frac{I_{440 \mathrm{~nm}}-I_{490 \mathrm{~nm}}}{I_{440 \mathrm{~nm}}+I_{490 \mathrm{~nm}}}
$$

Since Laurdan emission becomes redshifted in a polar environment, a decrease in the GP value indicates a more random state of the intercellular lipid layers, with polar solvent molecules penetrating into the skin structures. ${ }^{6}$

To implement multiphoton polarization and GP imaging in the study of intercellular lipid structures we used a setup similar to one previously described. ${ }^{7}$ The 780-nm output of a femtosecond Ti:sapphire laser was circularly polarized and used as the excitation source. The circularly polarized source was used to ensure equal excitation of Laurdan molecules inserted at different positions of the lipid layers. ${ }^{7}$ With a high-N.A. oil-immersion lens (Nikon S Fluor, 40×, N.A. 1.3), the laser power delivered to the sample varied from 2.8 to $3.3 \mathrm{~mW}$. These powers were determined to be below the signal saturation regime. To implement polarization microscopy we placed a sheet polarizer in front of the photomultiplier tube, and fluorescence images were acquired at crossed polarizations. The $I_{\text {par }}$ and $I_{\text {per }}$ axes were chosen to be horizontally and vertically oriented with respect to the acquired images. To implement GP imaging we switched 440-nm (HQ 440-40-m) and 490-nm (HQ 490-40-m) bandpass filters (both from Chroma Technology) to acquire spectrally resolved images used for GP analysis.

The sample used was excised human skin immersed in two types of labeling solution. In one sample the skin was immersed in a solution composed of $10 \mu \mathrm{l}$ of 2-mM Laurdan (6-dodecanoyl-2-dimethylaminonaphthalene, D-250, Molecular Probes) stock (in DMSO) and $1 \mathrm{~mL}$ of phosphate buffer saline (PBS). For the other sample the skin was immersed in a labeling solution composed of $10 \mu \mathrm{l}$ of the Laurdan stock, $53 \mu \mathrm{l}$ of $\mathrm{OA}$, and $1 \mathrm{ml}$ of $\mathrm{PBS}$. $\mathrm{OA}$ in the latter labeling solution is frequently used as a chemical enhancer in drug delivery studies. ${ }^{3,4}$ The incubation time for the skin specimens in both labeling solutions was $10.5 \mathrm{~h}$. After the incubation period, the skin was removed, rinsed in PBS, and wiped before it was mounted for viewing. Autofluorescence from the skin was estimated to be approximately 0.5 counts/pixel and was negligible compared with the Laurdan fluorescence level. If we define when the average fluorescence reaches 0.1 that of the surface intensity, then the penetration depth of Laurdan was 15 and $23 \mu \mathrm{m}$ in the presence and absence of $\mathrm{OA}$, respectively. To evaluate the effectiveness of polarization and GP imaging in detecting structural changes in the lipid layer we imaged the same area of the skin for polarization and GP imaging. The raw images acquired were then postprocessed [Eqs. (1) and (2)] to obtain polarization and GP images (Fig. 1). Histograms of the polarization and GP values are shown in Fig. 2. For the polarization calculations we also selected three regions in the images to calculate the average polarization values of each membrane orientation.
An examination of Fig. 1 shows interesting results. The polarization image in Fig. 1(a), without OA treatment, shows a more highly polarized map than Fig. 1(c), with OA treatment. Regions in Fig. 1(a) display both blue and green, whereas Fig. 1(c) shows a much more uniform distribution of the color distribution. To verify this observation we plotted the histograms of the polarization values and analyzed six regions with different lipid layer orientations (three vertical and three horizontal). These results are shown in Fig. 2. The average polarization values for Figs. 1(a) and 1(c) are $-0.01 \pm 0.18$ and $-0.02 \pm 0.16$, respectively. Therefore, although the polarization histograms did not show a significant difference between the two skin treatments, detailed analysis of the six regions clearly shows a difference in the polarization values. Figure 2(e) shows that the average polarization values for the horizontal and vertical orientations are $-0.09 \pm 0.08$ and $0.09 \pm 0.11$, respectively. $I_{\text {par }}$ and $I_{\text {per }}$ are defined to be horizontal and vertical, respectively, to the images shown. Therefore our polarization values are consistent with the observation that, without OA, the transition moments of the Laurdan molecules are preferentially oriented normal to the lipid surface. On the other hand, Fig. 2(f) shows that the polarization values of the skin sample treated with OA are $-0.01 \pm 0.08$ and $-0.01 \pm 0.08$, regardless of the lipid orientation.

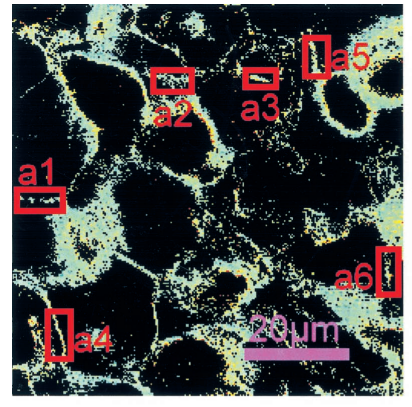

(a) P: without OA

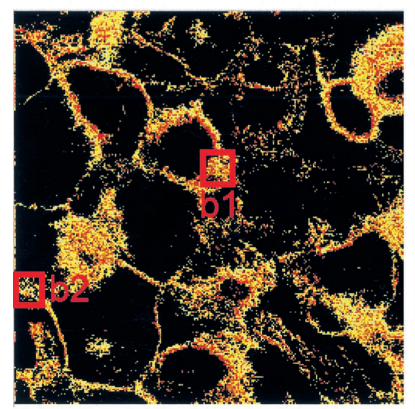

(b) GP: without OA

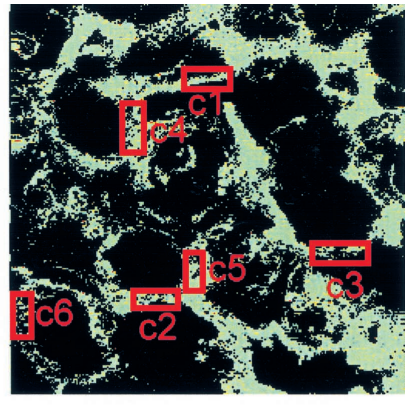

(c) P: with OA

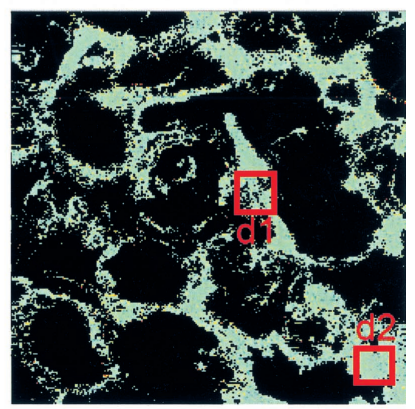

(d) GP: with OA

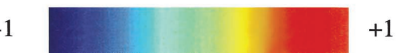

Fig. 1. Laurdan skin polarization images in the (a) absence and (c) presence of OA. Laurdan GP images (b) without and (d) with OA $\left(I_{\max }=1, I_{\min }=-1\right)$. In (b) and (d), two areas are chosen for GP analysis. In (b), the GPs for areas b1 and b2 are $0.37 \pm 0.15,0.27 \pm 0.14$, respectively. In (d) the GPs for $\mathrm{d} 1$ and $\mathrm{d} 2$ are, respectively, $-0.08 \pm 0.13$ and $-0.11 \pm 0.11$. 


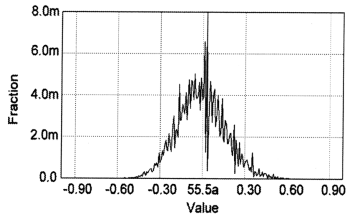

(a) P: without OA

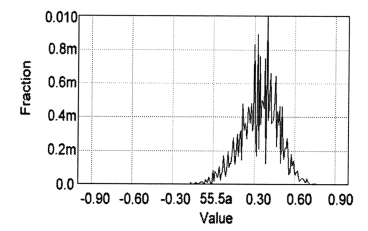

(b) GP: without OA

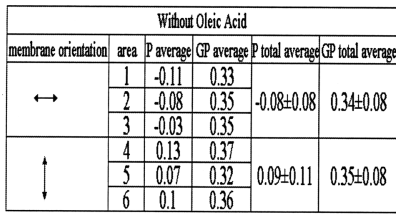

(e) P: without $\mathrm{OA}$ at selected regions

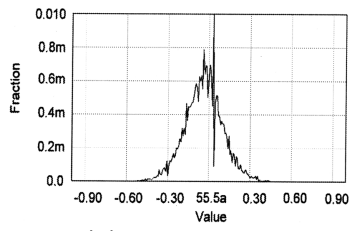

(c) P: with OA

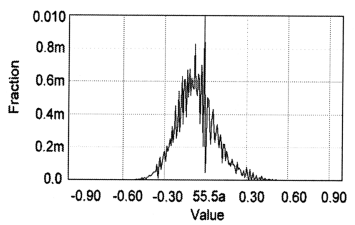

(d) GP: with OA

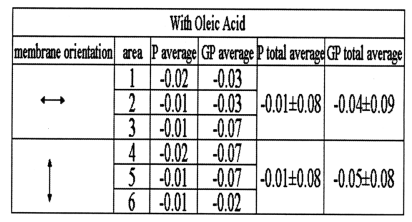

(f) P: with OA at selected regions
Fig. 2. Laurdan polarization histograms in the (a) absence and (c) presence of OA. (b) and (d) Laurdan GP histograms without and with OA, respectively. (e) and (f) Results of polarization and GP analysis without and with $\mathrm{OA}$, respectively, at selected regions.

Our results show that, although the effects of OA treatment on polarization images and histograms are not as apparent (because the polarization values are averaged over many different orientations), detailed image analysis of differently oriented lipid layers of the skin shows that the addition of OA tends to randomize the packing of lipid molecules in the skin. We also calculated the average GP values for the six regions investigated [Figs. 2(e) and 2(f)], and we found that regardless of the lipid orientation the GP decreases if $\mathrm{OA}$ is added. Our results suggest that OA perturbs the lipid packing and allows a more polar environment to exist in the lipids and that there is little correlation between polarization and GP values if $\mathrm{OA}$ is not added.

Also shown in Figs. 1 and 2 are the GP images and histograms of skin surface not treated and treated with OA. As indicated by Figs. 1(b) and 1(d), the skin treated with OA has a lower GP value. Figures 2(b) and 2(d) also show a clear shift of the GP to lower values if $\mathrm{OA}$ is added. We evaluated the average GP values across the entire image and found values of $0.35 \pm 0.14$ and $-0.06 \pm 0.15$ for skin treated without and with OA, respectively. Laurdan has been known to be a molecule whose GP value decreases in a more polarized environment. ${ }^{8}$ Therefore our GP results are consistent with the fact that the addition of OA disturbs the lipid packing in the skin and allows water molecules to penetrate the lipid layers of the skin. In addition, our data show that different regions of lipid fluidity may exist in the skin. An examination of Figs. 1(b) and 1(d) shows that without OA there are regions of different GP values. We selected two areas in the GP images, and Fig. 2(b) shows that without $\mathrm{OA}$ the GP values for $\mathrm{b} 1$ and $\mathrm{b} 2$ are $0.37 \pm 0.15$ and $0.27 \pm 0.14$, respectively. Figure $1(\mathrm{~d})$ shows that, if $\mathrm{OA}$ is added, the respective GP values of the two regions (d1 and $\mathrm{d} 2)$ are $-0.08 \pm 0.13$ and $-0.11 \pm 0.11$. Therefore, without the addition of OA, different areas of the skin can display local environments with different polarity, whereas OA tends to polarize the chemical environment of the skin.

In conclusion, we have demonstrated that polarization and generalized polarization imaging may be combined to monitor structural changes in the lipid structures of skin. Polarization imaging reveals the molecular orientation in biological structures, and GP imaging provides information on changes in the molecular microenvironment. A combination of multiphoton polarization and GP imaging is a useful technique for understanding physical and chemical changes in biological systems.

This work was supported by the National Science Council of Taiwan (grants NSC 92-2112-M-002-018 and NSC 92-3112-B-002-048). Correspondence in regard to this work should be addressed to Chen-Yuan Dong at cydong@phys.ntu.edu.tw.

\section{References}

1. W. Denk, J. H. Strickler, and W. W. Webb, Science 248, 73 (1990).

2. P. T. C. So, C. Y. Dong, B. R. Masters, and K. M. Berland, Ann. Rev. Biomed. Eng. 2, 399 (2000).

3. B. Yu, C. Y. Dong, P. T. C. So, D. Blankschtein, and R. Langer, J. Invest. Dermatol. 117, 16 (2001).

4. B. Yu, K. H. Kim, P. T. C. So, D. Blankschtein, and R. Langer, J. Invest. Dermatol. 118, 1085 (2002).

5. R. O. Potts and M. L. Francoeur, Proc. Natl. Acad. Sci. USA 87, 3871 (1990).

6. L. A. Bagatolli and E. Gratton, Biophys. J. 78, 290 (2000).

7. Y. Sun, J. W. Su, W. Lo, S. J. Lin, S. H. Jee, and C. Y. Dong, Opt. Express 11, 3377 (2003), http://www. opticsexpress.org.

8. J. A. Boustra, F. E. R. Dubbelaar, G. S. Gooris, and M. Ponec, Acta Derm. Venereol. Suppl. 208, 23 (2000).

9. K. C. Madison, J. Invest. Dermatol. 121, 231 (2003).

10. J. R. Lakowicz, Principles of Fluorescence Spectroscopy (Plenum, New York, 1999). 